\title{
LINK-CUTTING BUBBLES FOR THE STABILIZATION OF CONVECTION-DIFFUSION-REACTION PROBLEMS
}

\author{
F. BREZZI*, ${ }^{\dagger} \S$, G. HAUKE ${ }^{\ddagger}$, L. D. MARINI*, ${ }^{\dagger}$ and G. SANGALLI ${ }^{\dagger}$ \\ * Dipartimento di Matematica "F. Casorati", Università di Pavia, \\ Via Ferrata 1, 27100 Pavia, Italy \\ ${ }^{\dagger}$ I.M.A.T.I.-C.N.R., Via Ferrata 1, 27100 Pavia, Italy \\ ¥Departamento de Mecánica de Fluidos, Centro Politécnico Superior, \\ C/Maria de Luna 3, 50018 Zaragaza, Spain \\ §brezzi@imati.cnr.it
}

Received 30 August 2002

Communicated by N. Bellomo

\begin{abstract}
It is known that the addition and elimination of suitable bubble functions can result in a stabilized scheme of the SUPG-type. Residual-Free Bubbles (RFB), in particular, can assure a quasi-optimal stabilized scheme, but they are difficult to compute in one dimension and nearly impossible to compute in two and three dimensions, unless in special limit cases. Strongly convection-dominated problems (without reaction terms) are one of these cases, where it is possible to find reasonably simple computable bubbles that provide a stabilizing effect as good as that of true RFB. Here, although in a onedimensional framework, we analyze the case in which a non-negligible reaction term is present, and provide a simple recipe for spotting a suitable bubble space (adding two bubbles to each element) that provides a very good stabilizing effect. The method adapts very well to all regimes with continuous transitions from one regime to another. It is clear that the one-dimensional case, in itself, has no real interest. We believe, however, that the discussion can cast some light on the interaction between convection and reaction that could be useful in future works dealing with multidimensional, more realistic problems.
\end{abstract}

Keywords: Finite element methods; bubbles; stabilizations.

AMS Subject Classification: 65N12, 65N30, 76M10

\section{Introduction}

We present in this paper a new stabilization method for one-dimensional convectiondiffusion-reaction problems, particularly designed to treat the (most interesting) case of small diffusion, but is able to adapt naturally from the diffusion-dominated regime to the convection-dominated and/or to the reaction dominated regime in a very simple way. 
The case treated corresponds to that of the positive-definite operator, which physically corresponds to a dissipative or absorbing reacting term. Therefore, exponential boundary and internal layers can appear at the inflow or outflow boundaries and at the discontinuities of coefficients and forcing terms. It is known that, in the presence of under-resolved layers, the plain Galerkin method will produce oscillations that pollute the whole domain. Several approaches have been proposed to rectify this problem within the framework of Galerkin Finite Element Methods, mostly for advection-dominated equations.

The early attempts have been directed to change the shape of the test and/or trial functions (Petrov-Galerkin methods) in order to reproduce the classical upwind correction widely used in finite differences. Typically one used exponential functions that were local solutions of the homogeneous differential equation as test functions (see, for instance, Refs. 14, 21 and references therein) but it was far from clear how to adapt these ideas in multidimensional problem.

A much more general approach was found in the use of stabilized methods, where the variational formulation is augmented in order to gain control of the derivatives of the solution. Typical stabilized formulations are the SUPG method, ${ }^{9,10}$ and variations, such as GLS. ${ }^{18}$ The great advantage of this approach is its great generality, together with the fact that in many cases it is possible to analyze it and quasi-optimal error bounds are derived. ${ }^{19,20,22,25}$ Its main drawback resides in the presence af a stabilizing parameter that needs to be properly chosen. This problem was partly addressed in the variational multiscale method and the SGS method. ${ }^{17}$ Application of the SGS method to the advective-diffusive-reactive equation was explored in various papers (see, e.g. Refs. 15, 16 and references therein).

Another approach consists of enriching the finite element spaces by bubble functions. For advective-diffusive problems the relationships between the use of bubble functions and stabilized methods were first studied in Refs. 1 and 2. The possibility of using special bubbles (called Residual-Free-Bubbles or RFB) in order to find a suitable value for the stabilizing parameter in SUPG methods was first introduced in Ref. 8, and the equivalence of this approach with the variational multiscale method was pointed out in Ref. 3. The method also allows one to prove quasi optimal error bounds ${ }^{7,23}$ and can be generalized to a much wider variety of problems (see e.g. Ref. 12). Its main drawback is that it requires the solution of a local PDE. Although only a limited precision is needed in the solution of these local problems, a suitable strategy to obtain a cheap approximate solution has to be designed for each type of problem. ${ }^{8,6,11,5}$ Close connections were also found between the RFB approach and the earlier Petrov-Galerkin methods using exponential test functions. ${ }^{4,5}$

Yet another way of stabilizing the Galerkin method has been long pursued and defended by several authors. The idea was to stabilize by means of a proper choice of the grid. This includes the possibility of using a suitable refinement near the outflow boundary so that, actually, the stabilization is not needed anymore, like in the Shishkin meshes (see Ref. 24 and references therein). This also includes 
the simpler use of suitable nodes near the outflow boundary as advocated by Gresho and others ${ }^{13}$ : for instance for the one-dimensional convection-diffusion problem, the Galerkin method can be stabilized by the solely correct placement of the first node within the boundary layers of the flow. The drawback of these methodologies resides in that they require a priori knowledge of the flow physics and the placement of the boundary layers.

Finally, the method that is proposed in this paper emanates from merging the concepts of Gresho and bubble stabilization. Within each element, following Ref. 5 we choose a suitable subgrid (here made of two points), and we take the bubbles which are piecewise linear on the subgrid. The strategy for choosing the subgrid is as follows. Near each endpoint we look for the location of the node that would make the solution of the local RFB problem insensitive to the boundary value, as done by Gresho for advection-diffusion problems. If such position is close enough to the corresponding endpoint (here we took one third of the interval length) we take it, guessing that the local problem has a boundary layer. Otherwise, we put the node in a "neutral" position inside (the details will be given in Sec. 4). Hence we can perform our choice without requiring a priori knowledge of the flow physics.

The layout of the paper is the following. First, the augmented space strategy based on subgrid scales is reviewed in Sec. 2 and next particularized into two bubble functions in Sec. 3. The choice of the bubble functions and subgrid nodes is presented in Sec. 4 and the resulting method is analyzed for the various regimes in Sec. 5 .

\section{The Augmented Space Strategy}

We will consider, for the sake of simplicity, the following linear elliptic convectiondiffusion-reaction problem in an interval $I$ that, for simplicity, we scale to $I=]-1,1[$ :

$$
\mathcal{L} u=f \text { in } I, \quad u(-1)=u(1)=0,
$$

where

$$
\mathcal{L} u=-\varepsilon u^{\prime \prime}+\beta u^{\prime}+\sigma u .
$$

Let $\mathcal{T}_{h}=\{K\}$ be a decomposition of $I$ into subintervals $K$. For the sake of simplicity, we shall also assume that the decomposition is uniform, so that we can call $h$ the (common) length of the intervals in the subdivision. However, all our discussions will take place at the element level. Hence they will be valid for a general decomposition, just assuming that, in deciding what to do in a particular element, we take $h$ as the length of that element.

We assume that the diffusion coefficient $\varepsilon$ is a positive constant, and that the convection field $\beta$, the reaction field $\sigma$, and the right-hand side $f$ are piecewise constant with respect to the decomposition $\mathcal{T}_{h}$. We also assume that the reaction field $\sigma$ is non-negative, and that the convective field $\beta$ is nonincreasing and does not change sign (that means, more precisely, that we assume that either $\beta \geq 0$ 
in the whole $I$, or $\beta \leq 0$ in the whole $I$ ). So, unless $\beta \equiv 0$ (pure reaction case), we can speak of inflow and outflow. When $\varepsilon \ll|\beta| h+\sigma h^{2}$ the solution of the problem will (for a generic $f$ ) have boundary layers, that can be either "only at the outflow" (if any), or "at both ends of $I$ ", depending on the reciprocal values of $|\beta| h$ and $\sigma h^{2}$ (see also the discussion below). Internal layers may also occur (in the reaction-dominated regime, see Sec. 5.3). In these cases, the pure Galerkin method will typically fail, showing strong oscillations near the boundary layers, and some stabilization is needed.

Here we will consider stabilizations based on the augmented space idea, ${ }^{5}$ which includes the RFB strategy, and can be summarized as follows.

We start by recalling the classical variational formulation of problem (2.1):

$$
\left\{\begin{array}{l}
\text { find } u \in H_{0}^{1}(I) \text { such that } \\
a(u, v)=(f, v) \text { for all } v \in H_{0}^{1}(I)
\end{array}\right.
$$

where

$$
a(u, v)=\varepsilon \int_{I} u^{\prime} v^{\prime} d x+\int_{I}\left(\beta u^{\prime}\right) v d x+\int_{I} \sigma u v d x
$$

is a continuous and coercive bilinear form on the Hilbert space $H_{0}^{1}(I)$, and $(f, v)$ denotes the scalar product of $f$ and $v$ in $L^{2}(I)$. A Galerkin approximation of problem (2.1) consists of taking a finite-dimensional subspace $V_{h}$ of $H_{0}^{1}(I)$, and then solving the variational problem (2.3) in $V_{h}$. For the sake of simplicity, from now on we will restrict ourselves to the case of continuous, piecewise linear elements, i.e. we will consider the finite element space

$$
V_{L}=\left\{v \in H_{0}^{1}(i), v_{\mid K} \text { linear for all } K \in \mathcal{T}_{h}\right\}
$$

so that the approximation of (2.3) reads

$$
\left\{\begin{array}{l}
\text { find } u_{L} \in V_{L} \text { such that } \\
a\left(u_{L}, v_{L}\right)=\left(f, v_{L}\right) \text { for all } v_{L} \in V_{L} .
\end{array}\right.
$$

The idea behind the augmented space strategy is to enlarge the finite element space $V_{L}$ in the following way. For each element $K$, we define a suitable space of bubbles $B_{K} \subset H_{0}^{1}(K)$, to be chosen later on, and then we enlarge the space $V_{L}$ to

$$
V_{h}=V_{L} \oplus V_{B}, \quad \text { where } \quad V_{B}=\oplus_{K} B_{K} .
$$

The approximate problem in the enlarged space then reads

$$
\left\{\begin{array}{l}
\text { find } u_{h} \in V_{h} \text { such that } \\
a\left(u_{h}, v_{h}\right)=\left(f, v_{h}\right) \text { for all } v_{h} \in V_{h} .
\end{array}\right.
$$

The RFB method ${ }^{8,12}$ would correspond to the choice $B_{K} \equiv H_{0}^{1}(K)$, so that, in one dimension, $V_{h} \equiv H_{0}^{1}(I)$ and the solution in $V_{h}$ will actually be the exact solution. 
By (2.7) we have that any $v_{h} \in V_{h}$ can be split into a linear part $v_{L} \in V_{L}$ and a bubble part $v_{B} \in V_{B}$ in a unique way:

$$
v_{h}=v_{L}+v_{B} \in V_{L} \oplus V_{B},
$$

while the bubble part itself can be uniquely split element by element:

$$
v_{B}=\sum_{K} v_{B, K}, \quad v_{B, K} \in B_{K} .
$$

Then, the variational problem $(2.8)$ in $V_{h}$ can be written as follows:

$$
\left\{\begin{array}{l}
\text { find } u_{h}=u_{L}+u_{B} \in V_{L} \oplus V_{B} \text { such that } \\
a\left(u_{L}+u_{B}, v_{L}\right)=F\left(v_{L}\right) \quad \forall v_{L} \in V_{L} \text { and } \\
a\left(u_{L}+u_{B, K}, v_{B, K}\right)_{K}=\left(f, v_{B, K}\right)_{K} \quad \forall K \in \mathcal{T}_{h}, \quad \text { and } \forall v_{B, K} \in B_{K},
\end{array}\right.
$$

where the subscript $(\cdot)_{K}$ indicates that the integrals involved are restricted to the element $K$.

The second equation of (2.11) can, in each $K$, be written as

$$
a\left(u_{B, K}, v_{B, K}\right)_{K}=\left(f-\mathcal{L} u_{L}, v_{B, K}\right)_{K}, \quad \forall v_{B, K} \in B_{K} .
$$

We denote now by $M_{K}$ the linear operator that to every $g \in L^{2}(I)$ associates the unique solution $w=M_{K}(g) \in B_{K}$ of the problem

$$
a\left(w, v_{B, K}\right)_{K}=\left(g, v_{B, K}\right)_{K} \quad \forall v_{B, K} \in B_{K},
$$

so that (2.12) gives $u_{B, K}=M_{K}\left(f-\mathcal{L} u_{L}\right)$. Substituting $u_{B}=\sum u_{B, K}$ in the first equation of (2.11) we have then

$$
a\left(u_{L}, v_{L}\right)+\sum_{K} a\left(M_{K}\left(f-\mathcal{L} u_{L}\right), v_{L}\right)_{K}=\left(f, v_{L}\right) .
$$

We introduce now, for every $K$, the formal adjoint $\mathcal{L}_{K}^{*}$ of $\mathcal{L}$ on $K$, defined by $\mathcal{L}_{K}^{*} v=-\varepsilon v^{\prime \prime}-(\beta v)^{\prime}+\sigma v$, and we end up with the stabilized scheme:

$$
a\left(u_{L}, v_{L}\right)+\sum_{K}\left(M_{K}\left(\mathcal{L} u_{L}-f\right), \mathcal{L}_{K}^{*} v_{L}\right)_{K}=\left(f, v_{L}\right) \quad \forall v_{L} \in V_{L},
$$

where the term $\left(M_{K}\left(\mathcal{L} u_{L}-f\right), \mathcal{L}_{K}^{*} v_{L}\right)_{K}$ represents the (stabilizing) effect, on the linear scheme, of the bubble part.

Remark 1. As we already pointed out, by choosing $B_{K} \equiv H_{0}^{1}(I)$ as in the residualfree bubble method, the solution $u_{L}+u_{B}$ would coincide with the exact solution $u$, so that the linear part $u_{L}$ would be nodally exact. On the other hand, the solution of the local problems (2.12) would require the exact solution of a differential equation of the same type as the original one (2.1). Indeed, if $B_{K} \equiv H_{0}^{1}(I)$, then the local bubble problem (2.12) can also be written as

$$
\mathcal{L} u_{B, K}=f-\mathcal{L} u_{L} \quad \text { in } K
$$

which has clearly the same structure of (2.1). Notice in particular that from $(2.16)$ we immediately have that inside each element $K$ the solution $u_{h}=u_{B}+u_{L}$ satisfies $\mathcal{L} u_{h}=f$, whence the name Residual Free. 


\section{The Case of Two-Bubble Subgrid}

In this section we shall consider in more detail the case in which each bubble space $B_{K}$ is spanned by two functions, both piecewise linear inside $K$. To fix the ideas, we consider a typical element $K=] x_{1}, x_{2}\left[\right.$ (so that $h=x_{2}-x_{1}$ ). All the following discussion will take place in $K$, and therefore, at most occurrencies, we shall drop the index $K$ in the notation. In $K$ we choose two points, $z_{1}, z_{2}$, satisfying

$$
x_{1}<z_{1}<z_{2}<x_{2}
$$

that will define the subgrid in $K$. The criteria for the choice of $z_{1}$ and $z_{2}$ as functions of $\varepsilon, \beta, \sigma$ and $h$ will be given in the next section, together with the analysis of the corresponding scheme at the various regimes.

For the moment, we construct the bubble $b_{i},(i=1,2)$ as the function which is continuous and piecewise linear on the subgrid (3.1), has value 1 at $z_{i}$ and value 0 at the other points of the subgrid (3.1). We then set $B_{K}=\operatorname{span}\left\{b_{1}, b_{2}\right\}$. The corresponding local bubble problem (2.12) can now be written using as unknowns the values of $u_{B}$ at the points $z_{1}$ and $z_{2}$. Indeed, setting $u_{B}^{i}:=u_{B}\left(z_{i}\right)(i=1,2)$, we have from $(2.12)$

$$
\sum_{j=1}^{2} u_{B}^{j} a\left(b_{j}, b_{i}\right)_{K}=\left(f-\mathcal{L} u_{L}, b_{i}\right)_{K}, \quad i=1,2 .
$$

Clearly the operator $M_{K}$ defined in (2.13) can be identified with the inverse matrix of the subgrid stiffness matrix $S$ given by

$$
S_{i, j}:=a\left(b_{j}, b_{i}\right)_{K}
$$

Reintroducing in our notation the dependence on $K$ of the $u_{B}^{j}$ 's and of the functions $b_{j}$ 's, the final scheme can then be written as

$$
a\left(u_{L}, v_{L}\right)+\sum_{K} \sum_{j=1}^{2} u_{B, K}^{j}\left(b_{K, j}, \mathcal{L}_{K}^{*} v_{L}\right)_{K}=\left(f, v_{L}\right) \quad \forall v_{L} \in V_{L},
$$

where the $u_{B, K}^{j}$ 's are the solutions, in each $K$, of the problem (3.2), and therefore depend on $u_{L}$ and $f$. When analyzed for the particular choices of the subgrid, according to the different regimes, the formulation (3.4) will show in detail the stabilizing effect that our procedure has on the original piecewise linear scheme on the coarse grid.

There is, however, a different way of writing the augmented problem with the present choice of bubbles. Indeed we can consider an enriched grid $\mathcal{K}_{h}$ having both the original grid nodes and the added subgrid nodes all together. We can easily see that the augmented space $V_{h}$ is the space of piecewise linear continuous functions on $\mathcal{K}_{h}$. Hence, we might consider that we are just using plain Galerkin on the enriched grid $\mathcal{K}_{h}$. Then (in particular in two- and three-dimensional problems) we can still choose to perform a static condensation of the bubble degrees of freedom, but this time just for computational reasons. We can consider, in a sense, that the 
stabilizing effect is just due to the enrichment of the grid by means of a suitably chosen subgrid. ${ }^{5}$

In this case, we can think of a different representation of the stabilized scheme. Indeed, if we have in mind a solution $u_{h}$ that is piecewise linear on the enriched subgrid, it would not be convenient to present it in the splitting (2.9), (2.10). We can still, if we like, make a distinction between (i) the values of the discrete solution $u_{h}$ at the old nodes of the decomposition $\mathcal{T}_{h}$, and (ii) the values of $u_{h}$ at the new nodes added with the role of stabilizing subgrid. In the previous notation, these will be the values of $u_{h}$ at the nodes $x_{1}, x_{2}$, and at the nodes $z_{1}, z_{2}$, respectively. However, the former will still coincide with $u_{L}$, because, for instance, $u_{h}\left(x_{1}\right) \equiv u_{L}\left(x_{1}\right)$, as $u_{B}\left(x_{1}\right)$ will always be zero (it is a bubble!). But the latter will no longer be the $u_{B, K}^{j}$ 's: indeed, for instance, $u_{h}\left(z_{1}\right)=u_{L}\left(z_{1}\right)+u_{B}\left(z_{1}\right)$, and $u_{B}\left(z_{1}\right) \equiv u_{B, K}^{1}$, but $u_{L}\left(z_{1}\right)$ will not, in general, be zero (why should it?). In certain regimes, it will be much more convenient to analyze the schemes looking first at the values $u_{h}\left(z_{i}\right)$ of the full solution $u_{h}$ at the added (subgrid) nodes, and then looking at its values at the nodes of the original $\mathcal{T}_{h}$.

The two different approaches discussed above would correspond to two different choices for the basis of the augmented space $V_{h}$. Restricting to the interval $] x_{1}, x_{2}$ [ (i.e. to the grid (3.1)) we introduce the notation

$$
\begin{array}{ll}
v_{1}(x):=\left(x-x_{2}\right) /\left(x_{1}-x_{2}\right), & v_{2}(x):=\left(x-x_{1}\right) /\left(x_{2}-x_{1}\right), \\
\varphi_{1}(x):=\left[\left(x-z_{1}\right) /\left(x_{1}-z_{1}\right)\right]^{+}, & \varphi_{2}(x):=\left[\left(x-z_{2}\right) /\left(x_{2}-z_{2}\right)\right]^{+},
\end{array}
$$

(where, as usual, [ $]^{+}$represents the positive part); we see that the space of piecewise linear continuous functions on the grid (3.1) can be taken either as $\operatorname{span}\left\{v_{1}, b_{1}, b_{2}, v_{2}\right\}$ (first approach followed here) or as $\operatorname{span}\left\{\varphi_{1}, b_{1}, b_{2}, \varphi_{2}\right\}$ (second approach). Indeed, the first choice implies a splitting $u_{h}=u_{L}+u_{B}$, while the second one is basically like looking globally at the refined grid.

We shall see in the next section that both approaches are convenient, according to the different regimes.

\section{The Choice of the Subgrid Nodes}

As we have seen in the previous section, we decided to take two bubbles per element. The number of bubbles has been suggested by the dimension of the space spanned by the residual $\mathcal{L} u_{L}-f$ when $u_{L}$ is piecewise linear and $f$ piecewise constant. Moreover, the shape of the two subgrid bubbles in the typical interval ] $x_{1}, x_{2}$ [ has been chosen to be piecewise linear on the subgrid (3.1), and depends therefore only on the choice of the subgrid points $z_{1}, z_{2}$. Hence we are left with the problem of choosing $z_{1}, z_{2}$ as functions of the parameters $\varepsilon, \beta, \sigma$ and $h=x_{2}-x_{1}$, that is the size of the interval.

The main guideline for our choice will be the link-cutting strategy: the idea is that, when $\varepsilon$ is small (in a sense that we will make precise in a while) the bubble 
problem that would come out of the RFB approach (see (2.16))

$$
\left.\mathcal{L} u_{B}=f-\mathcal{L} u_{L} \quad \text { in }\right] x_{1}, x_{2}\left[, \quad \text { with } u_{B}\left(x_{1}\right)=u_{B}\left(x_{2}\right)=0\right.
$$

can have boundary layers. As we want to solve it, in an approximate way, on a subgrid having just two nodes, it seems convenient (and, in a sense, necessary) to "forget" the Dirichlet boundary condition whenever we have a boundary layer there. This, with the notation (3.5), amounts to choosing $z_{1}, z_{2}$ such that

- the coefficient $a\left(\varphi_{1}, b_{1}\right)=0$ when we have a boundary layer near $x_{1}$,

- the coefficient $a\left(\varphi_{2}, b_{2}\right)=0$ when we have a boundary layer near $x_{2}$.

Let us see how the above conditions can be enforced. We set

$$
\xi:=z_{1}-x_{1}, \quad \eta:=x_{2}-z_{2} \quad \text { and } \quad \delta:=z_{2}-z_{1} .
$$

To simplify the exposition, we shall assume, from now on, that the convection coefficient $\beta$ is $\geq 0$. It is clear that the whole discussion that follows will apply to the case $\beta \leq 0$ as well, just exchanging the roles of $x_{1}$ and $x_{2}$ (and consequently of $z_{1}$ and $z_{2}$ ). With an easy computation we see now that

$$
\begin{aligned}
& a\left(\varphi_{1}, b_{1}\right)=0 \leftrightarrow-\frac{\varepsilon}{\xi}-\frac{\beta}{2}+\frac{\sigma \xi}{6}=0 \leftrightarrow \xi=\frac{3 \beta+\sqrt{9 \beta^{2}+24 \varepsilon \sigma}}{2 \sigma} \\
& a\left(\varphi_{2}, b_{2}\right)=0 \leftrightarrow-\frac{\varepsilon}{\eta}+\frac{\beta}{2}+\frac{\sigma \eta}{6}=0 \leftrightarrow \eta=\frac{-3 \beta+\sqrt{9 \beta^{2}+24 \varepsilon \sigma}}{2 \sigma}
\end{aligned}
$$

where in both cases we took the positive root of the algebraic equation. We also point out that the final expression in (4.4) has to be changed into its limit $\eta=2 \varepsilon / \beta$ when $\sigma=0$, while all the equations in (4.3) will have no solution for $\sigma=0$ (in a sense, they give $\xi=+\infty$ for $\sigma=0)$. Hence we set

$$
\begin{aligned}
& \xi_{e}:=\frac{3 \beta+\sqrt{9 \beta^{2}+24 \varepsilon \sigma}}{2 \sigma} \text { for } \sigma \neq 0 \text { and } \xi_{e}:=+\infty \text { for } \sigma=0, \\
& \eta_{e}:=\frac{-3 \beta+\sqrt{9 \beta^{2}+24 \varepsilon \sigma}}{2 \sigma} \text { for } \sigma \neq 0 \text { and } \eta_{e}:=2 \varepsilon / \beta \text { for } \sigma=0 .
\end{aligned}
$$

It is also clear that the values $\xi=\xi_{e}$ and $\eta=\eta_{e}$ dictated by (4.3) and (4.4) cannot be taken as such, in a blind way, for all regimes. To start with, from (4.2) and (3.1) we see that we have to require $\xi+\eta<h$. Hence we are going to need a cutoff in the choice of $\xi$ and $\eta$, whenever $\xi_{e}$ and $\eta_{e}$ are too large. At the same time, we do not want $\delta$ to be too small either (when compared with $\xi$ and $\eta$ ). For simplicity we can decide that we want, say,

$$
\delta \geq \min \{\xi, \eta\}
$$

We also notice that we always have $\eta_{e} \leq \xi_{e}$ (from (4.5) and (4.6)). As our strategy will be of cutoff type, we can decide to choose $\eta \leq \xi$, so that (4.7) becomes $\delta \geq \eta$. 
Hence $\eta$ will always be the smallest of the three sublengths. As the sum of the three is $h$, we end up with the (very reasonable) condition

$$
\eta \leq h / 3
$$

We notice now that $\eta_{e}$, as given by (4.6), will always tend to 0 when $\varepsilon \rightarrow 0$ (unless both $\beta$ and $\sigma$ are zero, a case that we already discarded). In particular we shall have that (4.8) holds true whenever

$$
6 \varepsilon \leq \beta h+\sigma h^{2} / 9 .
$$

This is the first "bifurcation" in our strategy. Indeed, if (4.9) does not hold, we consider that our problem (in the given interval) is diffusion dominated, and we set $\eta=h / 3$ (which implies then $\xi=\delta=h / 3$ as well). Hence:

- if $6 \varepsilon \geq \beta h+\sigma h^{2} / 9$, then $\eta=h / 3$.

The interesting cases, however, occur when (4.9) does hold. In that case, we take for $\eta$ the value coming out of (4.4), that is $\eta_{e}$ given by (4.6):

- if $6 \varepsilon \leq \beta h+\sigma h^{2} / 9$ then $\eta=\eta_{e}$.

We made our choice for $\eta$. It remains to choose $\xi$. Applying (4.7) we see that we cannot choose $\xi>h-2 \eta$, otherwise $\delta \equiv h-\xi-\eta<\eta$. Hence

- in all cases we take $\xi=\min \left\{h-2 \eta, \xi_{e}\right\}$,

where $\xi_{e}$ is given by (4.5).

\section{Analysis of the Scheme in Various Regimes}

We have now fully defined our subgrid scheme. The corresponding subgrid stiffness matrix introduced in (3.3) can be specialized as

$$
\begin{aligned}
S_{1,1} & =\frac{\varepsilon}{\xi}+\frac{\sigma \xi}{3}+\frac{\varepsilon}{\delta}+\frac{\sigma \delta}{3}, & S_{1,2} & =-\frac{\varepsilon}{\delta}+\frac{\beta}{2}+\frac{\sigma \delta}{6} \\
S_{2,1} & =-\frac{\varepsilon}{\delta}-\frac{\beta}{2}+\frac{\sigma \delta}{6}, & S_{2,2} & =\frac{\varepsilon}{\delta}+\frac{\sigma \delta}{3}+\frac{\varepsilon}{\eta}+\frac{\sigma \eta}{3} .
\end{aligned}
$$

In order to study the behavior of the scheme at various regimes, we will follow the steps of our algorithm.

\subsection{Diffusion dominated regime}

In our algorithm, this will occur when $\eta_{e}>\eta=h / 3$, that is when $6 \varepsilon>\beta h+\sigma h^{2} / 9$. Our choices imply $\xi=\delta=\eta=h / 3$. Indeed, in this regime, the stabilization is not needed, and a uniform subgrid seems appropriate. 


\subsection{Convection dominated regime}

This will occur when $\eta_{e}=\eta \leq h / 3$ and $\xi_{e}>\xi=h-2 \eta$. In this case we will also have $\delta=\eta=\eta_{e}$, so that the subgrid stiffness matrix (5.1) will verify

$$
S_{1,2} \equiv a\left(b_{2}, b_{1}\right) \equiv-\frac{\varepsilon}{\delta}+\frac{\beta}{2}+\frac{\sigma \delta}{6} \equiv-\frac{\varepsilon}{\eta}+\frac{\beta}{2}+\frac{\sigma \eta}{6}=0 .
$$

More precisely, we have

$$
\begin{aligned}
S_{1,1} & =\frac{\varepsilon}{\xi}+\frac{\sigma \xi}{3}+\frac{\varepsilon}{\eta}+\frac{\sigma \eta}{3}, & S_{1,2} & =0 \\
S_{2,1} & =-\beta, & S_{2,2} & =\frac{2 \varepsilon}{\eta}+\frac{2 \sigma \eta}{3} .
\end{aligned}
$$

We can solve the local system (3.2) and insert the solution into (3.4). The stabilizing term, to be added to the original scheme as in (2.15), becomes now

$$
\frac{R_{1}}{S_{1,1}}\left(b_{1}, \mathcal{L}^{*} v_{L}\right)+\left(\frac{R_{2}}{S_{2,2}}-\frac{S_{2,1} R_{1}}{S_{1,1} S_{2,2}}\right)\left(b_{2}, \mathcal{L}^{*} v_{L}\right),
$$

where $R_{i}:=\left(f-\mathcal{L} u_{L}, b_{i}\right)(i=1,2)$. We can now analyze the resulting scheme in the limit for $\varepsilon \rightarrow 0$. Assuming, for simplicity, that $3 \beta>\sigma h$, it is not difficult to see that the problem will stay in the convection-dominated regime for all $\varepsilon$. Since, for $\varepsilon$ small, $\delta=\eta \simeq 2 \varepsilon / \beta$, we easily see that for $\varepsilon \rightarrow 0$

$$
\xi \rightarrow h, \quad S_{1,1} \rightarrow \frac{\sigma h}{3}+\frac{\beta}{2}, \quad S_{2,2} \rightarrow \beta
$$

and

$$
b_{1} \rightarrow v_{2}, \quad b_{2} \rightarrow 0 \quad \text { in } L^{2}(I),
$$

where $v_{2}=\left(x-x_{1}\right) / h$ is the basis function defined in (3.5). Consequently, $R_{2} \rightarrow 0$, $\left(b_{2}, \mathcal{L}^{*} v_{L}\right) \rightarrow 0$, and $R_{1} \rightarrow\left(f-\mathcal{L} u_{L}, \mathcal{L}^{*} v_{2}\right)$, so that $(5.4)$ becomes

$$
\frac{6}{2 \sigma h+3 \beta} \int_{K}\left(f-\mathcal{L} u_{L}\right) v_{2} d x \int_{K} v_{2} \mathcal{L}^{*} v_{L} d x .
$$

Notice that this is the same limit that we would get by using a single bubble, piecewise linear on the subgrid $x_{1}<z_{2}<x_{2}$. Hence, in this regime, and for $\varepsilon$ "small enough", a single bubble (suitably located) will suffice.

From (5.7) it is not difficult to see that, for the case $\sigma=0$ and $f$ constant, the stabilizing term (5.7) coincides with that given by the SUPG scheme:

$$
\tau_{K} \int_{K}\left(f-\mathcal{L} u_{L}\right) \mathcal{L}^{*} v_{L} d x
$$

with $\tau_{K}=h /(2 \beta)$.

In Fig. 1 we present the coarse-grid numerical solution $u_{L}$, the refined-grid numerical solution $u_{h}$ and the exact solution $u$ when $\varepsilon=10^{-2}, \beta=1, \sigma=1, f=1$ and $h=0.2$, i.e. ten elements are considered. In Fig. 2 the results for $\varepsilon=10^{-5}$ are reported. Figure 3 shows the $L^{1}$ relative errors for $u-u_{L}$ versus $h$; the behavior is $O(h)$, as expected, also for large $h$; the change of slope in the case $\varepsilon=10^{-2}$ corresponds to the transition to the diffusion-dominated regime. 


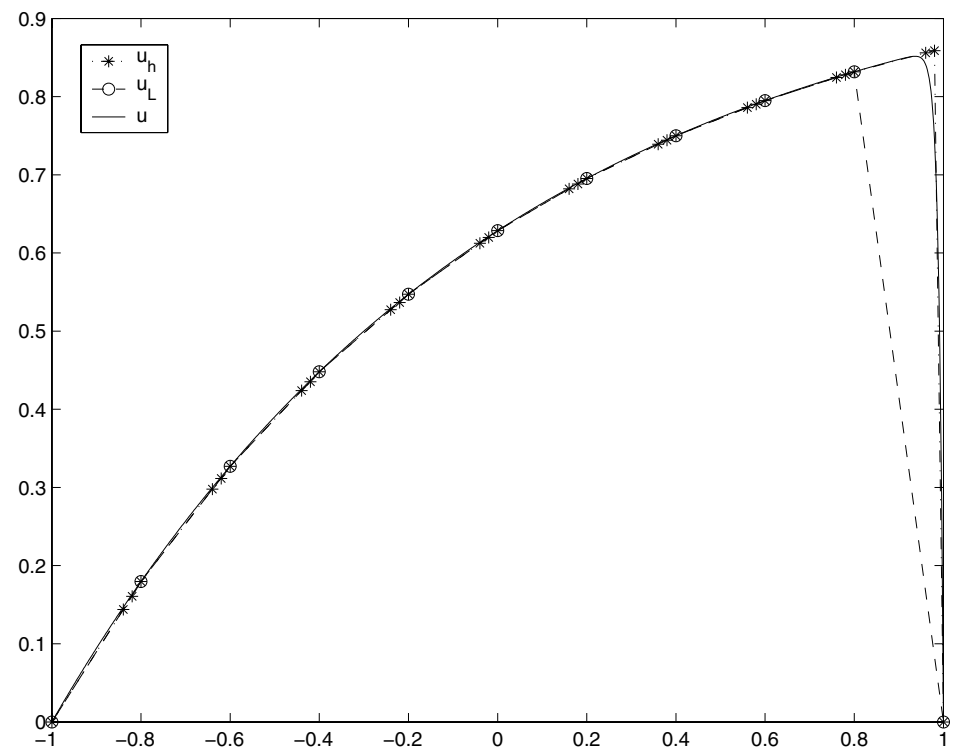

Fig. 1. Convection dominated regime: $\varepsilon=10^{-2}$.

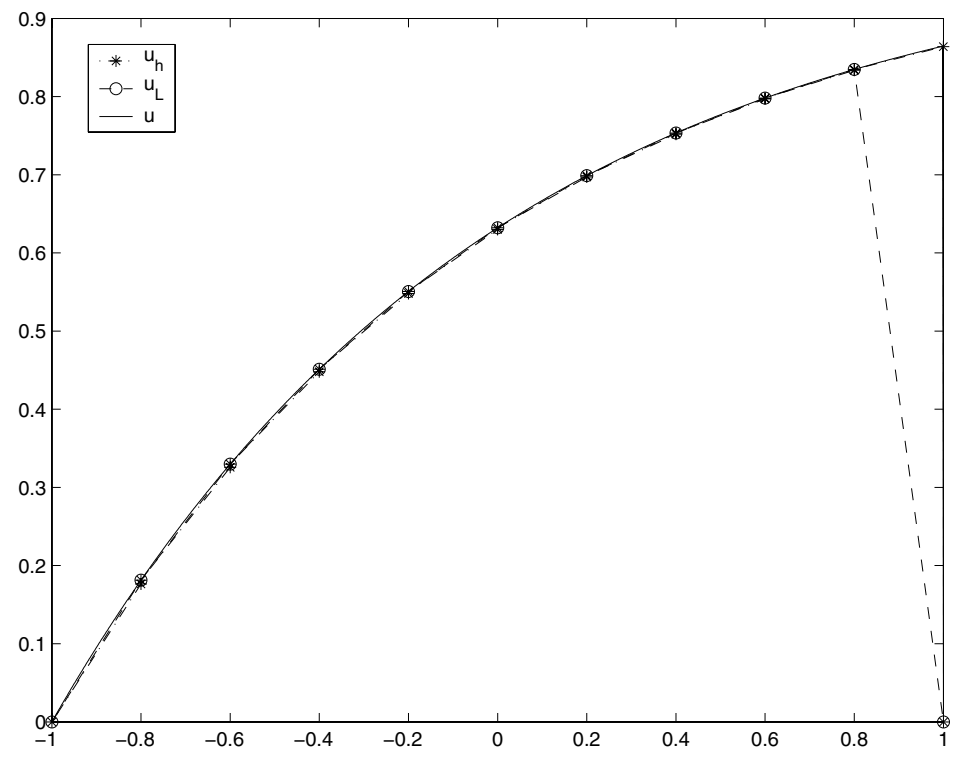

Fig. 2. Convection dominated regime: $\varepsilon=10^{-5}$.

\subsection{Reaction-dominated regime}

This will occur when $\eta_{e}=\eta \leq h / 3$ and $\xi_{e}=\xi \leq h-2 \eta$. In this regime, we have both $\xi=\xi_{e}$ and $\eta=\eta_{e}$ so that our link-cutting strategy will be fully operational, 


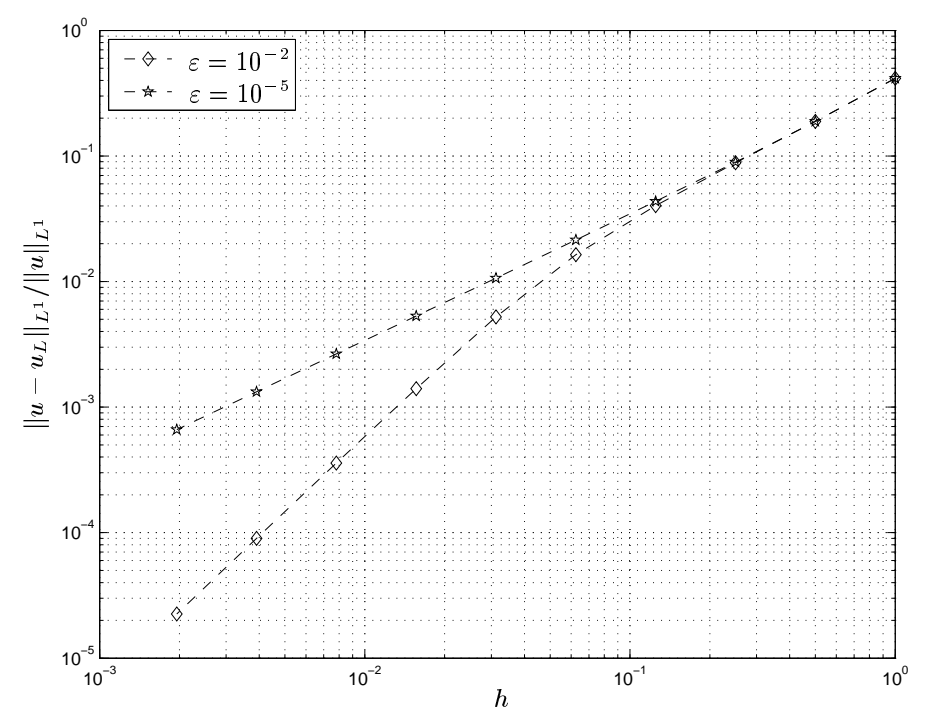

Fig. 3. $L^{1}$-error versus $h$ for the test problems of Figs. 1 and 2 .

and in particular we have both

$$
a\left(\varphi_{1}, b_{1}\right)=0 \text { and } a\left(\varphi_{2}, b_{2}\right)=0 .
$$

In order to analyze the scheme, it will now be more convenient to study directly the resulting scheme on the refined grid $\mathcal{K}_{h}$, i.e. (2.8). Indeed from (5.9) we have that the values of $u_{h}$ at the subgrid points $z_{i}$ will solve the following problem, (which is obtained by setting $v_{h}=b_{1}$ and then $v_{h}=b_{2}$ in the refined-grid problem (2.8)):

$$
\begin{aligned}
& S_{1,1} u_{h}\left(z_{1}\right)+S_{1,2} u_{h}\left(z_{2}\right)=\left(f, b_{1}\right), \\
& S_{2,1} u_{h}\left(z_{1}\right)+S_{2,2} u_{h}\left(z_{2}\right)=\left(f, b_{2}\right),
\end{aligned}
$$

with $S_{i, j}$ given by (5.1). Hence, the solution at the subgrid nodes can be computed by means of as many $2 \times 2$ systems as the number of intervals in the coarse grid. Once the value of the solution at the subgrid points has been computed, we can compute the value of the solution at the points of the original coarse grid $\mathcal{T}_{h}$ by solving a diagonal system, whose right-hand side depends only on $f$ and on the values of the solution at the subgrid points.

Before performing the analysis, it will be convenient, for notational purposes, to set $g:=f / \sigma$. In our case $g$ is hence assumed piecewise constant, therefore constant on $K=] x_{1}, x_{2}[$, and one can easily get

$$
a\left(g, b_{i}\right)=\int_{x_{1}}^{x_{2}} \sigma g b_{i}=\left(f, b_{i}\right), \quad i=1,2 .
$$

From (5.9) we also have $a\left(g, b_{i}\right)=g a\left(1, b_{i}\right)=g a\left(\varphi_{1}+b_{1}+b_{2}+\varphi_{2}, b_{i}\right)=g a\left(b_{1}+\right.$ $\left.b_{2}, b_{i}\right)=g\left(S_{i, 1}+S_{i, 2}\right)$, using also the fact that $a\left(\varphi_{1}, b_{2}\right)=a\left(\varphi_{2}, b_{1}\right)=0$, since 
the supports intersect only in one point. This, together with (5.11), inserted in (5.10) gives

$$
u_{h}\left(z_{1}\right)=u_{h}\left(z_{2}\right)=g
$$

The general idea behind (5.12) is that any linear function satisfying the differential equation in the element coincides with $u_{h}$ on the subgrid nodes. Indeed, if $g$ were linear, one could still apply this reasoning: " $g-\beta g^{\prime} / \sigma$ is a solution on the element of the differential equation, and therefore $u_{h}\left(z_{i}\right)=g\left(z_{i}\right)-\beta g^{\prime} / \sigma^{\prime}$.

If we want now to evaluate $u_{h}=u_{L}$, for instance, at the coarse node $x_{2}$, we have to use the information from the two intervals having $x_{2}$ in common, say $K^{1}:=K=$ ]$x_{1}, x_{2}\left[\right.$ and $\left.K^{\mathrm{r}}:=\right] x_{2}, x_{3}$ [. In the sequel the superscript indices "r" and "l" will refer to the "right" and "left" element respectively. We also extend our notation to $K^{\mathrm{r}}$ : e.g. $z_{3}<z_{4}$ will denote the two subgrid nodes, $b_{3}$ and $b_{4}$ the corresponding basis functions, and so on. We know that $u_{h}\left(x_{2}\right)=u_{L}\left(x_{2}\right)$ only depends on $f^{\mathrm{l}}, f^{\mathrm{r}}$ and $u_{h}\left(z_{i}\right), i=1, \ldots, 4$, which, as we have seen, depend only on $f^{\mathrm{l}}$ and $f^{\mathrm{r}}$ as well. Indeed, using (5.12) on both $K^{1}$ and $K^{\mathrm{r}}$, the equation for $u_{h}\left(x_{2}\right)$ reads:

$$
\begin{aligned}
u_{h}\left(x_{2}\right) a\left(\varphi_{2}, \varphi_{2}\right) & =\left(f, \varphi_{2}\right)-u_{h}\left(z_{2}\right) a\left(b_{2}, \varphi_{2}\right)-u_{h}\left(z_{3}\right) a\left(b_{3}, \varphi_{2}\right) \\
& =\left(f, \varphi_{2}\right)-g^{1} a\left(b_{2}, \varphi_{2}\right)-g^{\mathrm{r}} a\left(b_{3}, \varphi_{2}\right) .
\end{aligned}
$$

The final expression for $u_{h}\left(x_{2}\right)$, solution of (5.13), will in general depend on $h$, on $g$, and on the coefficients of the differential operator; in any case, however, $u_{h}\left(x_{2}\right)$ will stay in between $u_{h}\left(z_{2}\right)$ and $u_{h}\left(z_{3}\right)$, as we are now going to show. For this, we analyze first the contributions to (5.13) coming from $K^{1}$ : reasoning as in (5.11) yields

$$
a\left(g^{1}, \varphi_{2}\right)_{K^{1}}=\left(f^{1}, \varphi_{2}\right)_{K^{1}},
$$

and then, using the fact that $1-b_{2}=\varphi_{2}$ in $K^{1} \cap \operatorname{supp}\left\{\varphi_{2}\right\}$,

$$
\left(f^{1}, \varphi_{2}\right)_{K^{1}}-g^{1} a\left(b_{2}, \varphi_{2}\right)=g^{1}\left(a\left(1, \varphi_{2}\right)_{K^{1}}-a\left(b_{2}, \varphi_{2}\right)\right)=g^{1} a\left(\varphi_{2}, \varphi_{2}\right)_{K^{1}} .
$$

A result similar to (5.15) clearly holds for $K^{\mathrm{r}}$. Inserting into (5.14) we have

$$
u_{h}\left(x_{2}\right) a\left(\varphi_{2}, \varphi_{2}\right)=g^{\mathrm{l}} a\left(\varphi_{2}, \varphi_{2}\right)_{K^{1}}+g^{\mathrm{r}} a\left(\varphi_{2}, \varphi_{2}\right)_{K^{\mathrm{r}}} .
$$

We check now that both "weights" $a\left(\varphi_{2}, \varphi_{2}\right)_{K^{1}}$ and $a\left(\varphi_{2}, \varphi_{2}\right)_{K^{\mathrm{r}}}$ are positive. This is obvious for the first one. For the second one, using (4.3), we have

$$
a\left(\varphi_{2}, \varphi_{2}\right)_{K^{\mathrm{r}}}=\frac{\varepsilon}{\xi^{\mathrm{r}}}-\frac{\beta^{\mathrm{r}}}{2}+\frac{\sigma^{\mathrm{r}} \xi^{\mathrm{r}}}{3}=\frac{2 \varepsilon}{\xi^{\mathrm{r}}}+\frac{\sigma^{\mathrm{r}} \xi^{\mathrm{r}}}{6}>0 .
$$

As both weights are positive, (5.16) implies that $u_{h}\left(x_{2}\right)$ is between $g^{\mathrm{l}}$ and $g^{\mathrm{r}}$. When $\beta=0$ and $\sigma^{\mathrm{l}}=\sigma^{\mathrm{r}}$, one gets $u_{h}\left(x_{2}\right)=\left(u_{h}\left(z_{2}\right)+u_{h}\left(z_{3}\right)\right) / 2$, as expected by symmetry.

We can summarize the properties of the proposed scheme in the reaction dominated regime as follows: away from layers, $u_{h}$ is a very accurate approximation of $u \approx g \equiv f / \sigma$; the boundary layers are not seen by the method, while the internal layers, due to possible discontinuities in $f / \sigma$ and located across the coarse grid nodes, do not produce spurious oscillations. 
In Fig. 4 we present the coarse-grid numerical solution $u_{L}$, the refined-grid numerical solution $u_{h}$ and the exact solution $u$ for $\varepsilon=10^{-2}, \beta=1, \sigma=50$, $f=50 \cdot \operatorname{sign}(x)$ and $h=0.2$. In Fig. 5 the results for $\varepsilon=10^{-5}$ are reported. Figure 6 shows the $L^{1}$ relative errors for $u-u_{L}$ versus $h$; the same considerations as for Fig. 3 hold.

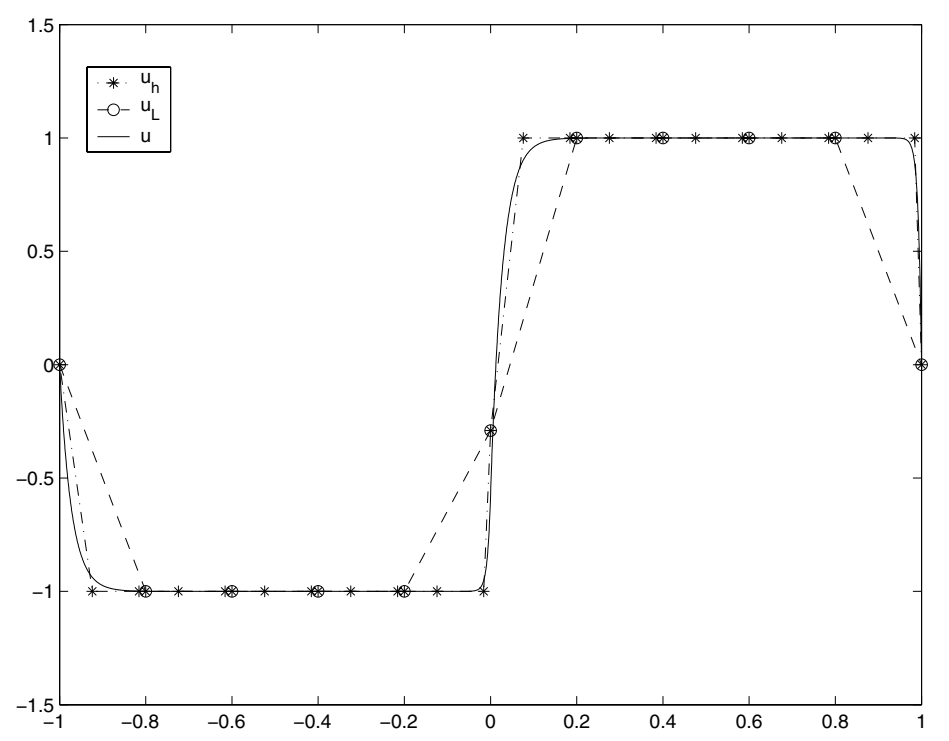

Fig. 4. Reaction-dominated regime: $\varepsilon=10^{-2}$.

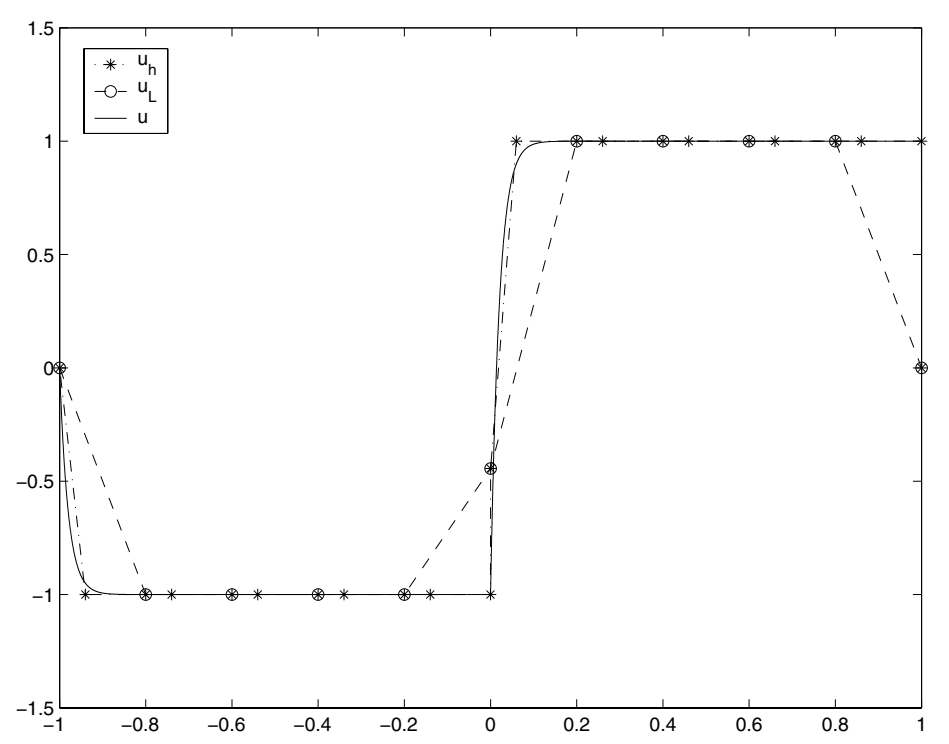

Fig. 5. Reaction-dominated regime: $\varepsilon=10^{-5}$. 


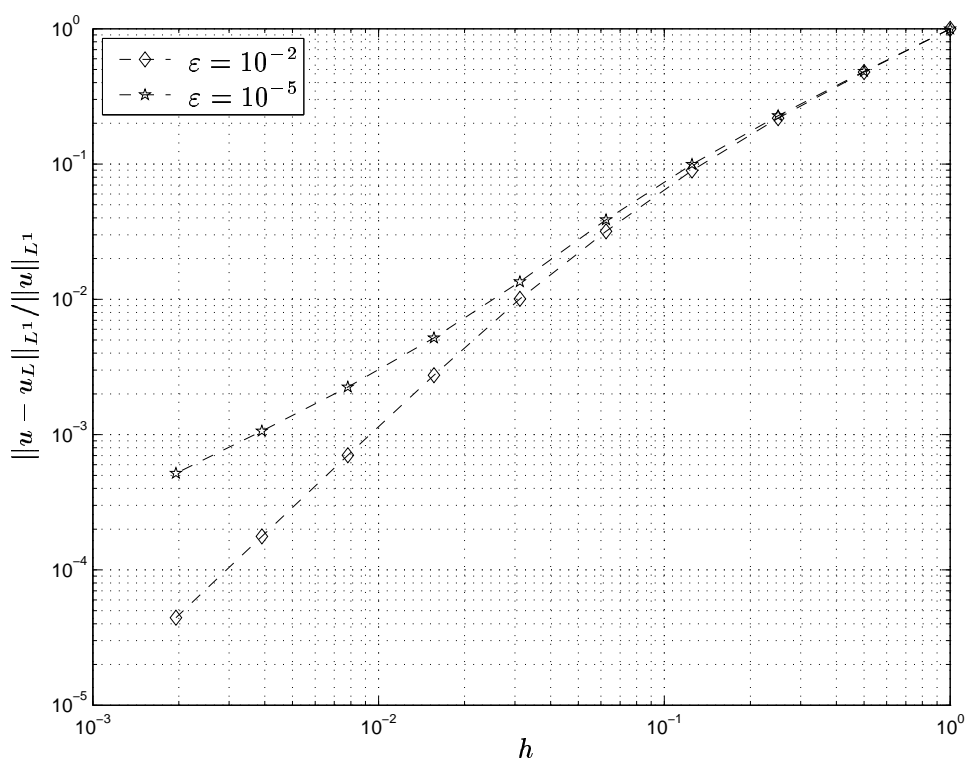

Fig. 6. $\quad L^{1}$-error versus $h$ for the test problems of Figs. 4 and 5 .

\section{Conclusions}

We wanted to stabilize a problem having both convection and reaction terms (and a small diffusion coefficient), starting from an original discretization with piecewise linear continuous finite elements, and an original mesh that was not especially suited to the problem (for instance, a uniform decomposition). In particular we wanted to mimic the stabilizing effect of Residual Free Bubbles, without actually computing them (that, in more than one dimension, would be unfeasible). As the residual in each element is a polynomial of degree $\leq 1$, we took two bubbles per element. In dimension $n$ we would need $n+1$ bubbles per element, which is still affordable. We opted for bubbles that are themselves piecewise linear, but on a suitable subgrid. As we know, the choice of the shape of the bubbles is crucial in determining the stabilizing effect of the final scheme. Here, with our premises, choosing the shape of the bubbles means choosing the subgrid in each element, so that, actually, we can treat the resulting scheme as doing plain Galerkin on the new, finer grid that results adding the element subgrid to the original one.

The basic idea behind our choice of the subgrid has been the link cutting strategy, which can be seen as an extension of a very old idea applied (for purely convection-diffusion problems) in order to have good results adding just one node suitably close to the outflow boundary. Here we can have two boundary layers, plus some internal ones due to possible jumps in the coefficients. Besides, we wanted a strategy that can operate without any a priori knowledge of the flow physics. Hence, we added two nodes per element. 
The location of our nodes depends on the regime that we have in each subinterval $K$, that can be determined by looking only at the coefficients of the local stiffness matrix. If we are diffusion-dominated, we just take equally spaced nodes (at $1 / 3$ and $2 / 3$ of the length of $K$ ). If convection dominates (in a sense that has been made precise) we have one node close to the outflow boundary of $K$, and the other close to it. When reaction takes the lead (again, in a sense that has been made precise) the second node drifts continuously toward the other endpoint of $K$, and for a pure reaction-diffusion problem we have two points that are symmetrically close to the two endpoints of $K$. The transition from one regime to another is continuous.

In the paper we described the formulas that rule the above choices, and we analyzed the resulting schemes, with particular attention to the case of a very small diffusion coefficient, and to its two subcases of strongly convection-dominated and strongly reaction-dominated schemes.

It does not seem science fiction to hope to reproduce a similar strategy in more than one dimension, and this makes, in our opinion, the main interest of the paper.

\section{References}

1. C. Baiocchi, F. Brezzi and L. P. Franca, Virtual bubbles and the GaLS, Comput. Methods Appl. Mech. Engrg. 105 (1993) 125-141.

2. F. Brezzi, M. O. Bristeau, L. P. Franca, M. Mallet and G. Rogé, A relationship between stabilized finite element methods and the Galerkin method with bubble functions, Comput. Methods Appl. Mech. Engrg. 96 (1992) 117-129.

3. F. Brezzi, L. P. Franca, T. J. R. Hughes and A. Russo, $b=\int g$, Comput. Methods Appl. Mech. Engrg. 142 (1997) 353-360.

4. F. Brezzi and L. D. Marini, Subgrid phenomena and numerical schemes, in Mathematical Modeling and Numerical Simulation in Continuum Mechanics, eds. I. Babuska, P. G. Ciarlet and T. Miyoshi, Springer Lect. Notes in Comput. Sci. Engrg., Vol. 19 (Springer, 2002), pp. 73-90.

5. F. Brezzi and L. D. Marini, Augmented spaces, two-level methods, and stabilising subgrids, Int. J. Numer. Meth. Fluids 40 (2002) 31-46.

6. F. Brezzi, L. D. Marini and A. Russo, Applications of pseudo residual-free bubbles to the stabilization of convection-diffusion problems, Comput. Methods Appl. Mech. Engrg. 166 (1998) 51-63.

7. F. Brezzi, L. D. Marini and E. Süli, Residual-free bubbles for advection-diffusion problems: The general error analysis, Numer. Math. 85 (2000) 31-47.

8. F. Brezzi and A. Russo, Choosing bubbles for advection-diffusion problems, Math. Models Methods Appl. Sci. 4 (1994) 571-587.

9. A. N. Brooks and T. J. R. Hughes, Streamline upwind/Petrov-Galerkin formulations for convection dominated flows with particular emphasis on the incompressible NavierStokes equations, Comput. Methods Appl. Mech. Engrg. 32 (1982) 199-259.

10. L. P. Franca, S. L. Frey and T. J. R. Hughes, Stabilized finite element methods: I. Application to the advective-diffusive model, Comput. Methods Appl. Mech. Engrg. $\mathbf{9 5}$ (1992) 253-276.

11. L. P. Franca, A. Nesliturk and M. Stynes, On the Stability of RFB for convectiondiffusion problems and their approximation by a two-level FEM, Comput. Methods Appl. Mech. Engrg. 166 (1998) 35-49. 
12. L. P. Franca and A. Russo, Deriving upwinding, mass lumping and selective reduced integration by RFB, Appl. Math. Lett. 9 (1996) 83-88.

13. P. M. Gresho and R. L. Sani, Incompressible Flow and the Finite Element Method (Wiley, 1998), Vol. 1.

14. D. F. Griffiths and J. Lorenz, An analysis of the Petrov-Galerkin method, Comput. Methods Appl. Mech. Engrg. 14 (1978) 39-64.

15. G. Hauke, A simple stabilized method for the advection-diffusion-reaction equation, Comput. Methods Appl. Mech. Engrg. 191 (2002) 2925-2947.

16. G. Hauke and A. Garcia-Olivares, Variational subgrid scale formulations for the advection-diffusion-reaction equation, Comput. Methods Appl. Mech. Engrg. 190 (2001) 6847-6865.

17. T. J. R. Hughes, Multiscale phenomena: Green's functions, the Dirichlet-to-Neumann formulation, subgrid scale models, bubbles and the origin of stabilized methods, Comput. Methods Appl. Mech. Engrg. 127 (1995) 387-401.

18. T. J. R. Hughes, L. P. Franca and G. Hulbert, A new finite element formulation for computational fluid dynamics: VIII. The Galerkin/least-squares method for advectivediffusive equations, Comput. Methods Appl. Mech. Engrg. 73 (1989) 173-189.

19. C. Johnson, U. Nävert, and J. Pitkäranta, Finite element methods for linear hyperbolic problems, Comput. Methods Appl. Mech. Engrg. 45 (1984) 285-312.

20. C. Johnson, A. H. Schatz and L. B. Wahlbin, Crosswind smear and pointwise error estimates in streamline diffusion finite element method, Math. Comp. 49 (1987) 25-38.

21. A. R. Mitchell and D. F. Griffiths, Generalised Galerkin methods for second order equations with significant first derivative terms, in Proc. Biennial Conf. Numer. Anal., Dundee 1977, Lect. Notes Math., Vol. 630 (Springer, 1978), pp. 90-104.

22. H.-G. Roos, M. Stynes and L. Tobiska, Numerical Methods for Singularly Perturbed Differential Equations: Convection Diffusion and Flow Problems (Springer-Verlag, 1996).

23. G. Sangalli, Global and local error analysis for the residual-free bubbles method applied to advection-dominated problems, SIAM J. Numer. Anal. 38 (2000) 1496-1522.

24. P. A. Farrell, A. F. Hegarty, J. J. H. Miller, E. O'Riordan and G. I. Shishkin, Robust computational techniques for boundary layers, Appl. Math. 16 (2000).

25. G. H. Zhou and R. Rannacher, Pointwise superconvergence of the streamline diffusion FEM, Numer. Methods for PDE 12 (1996) 123-145. 